\title{
OTHONOMTCS
}

Revista de los Estudios de Economía y Empresa

Dossier «Redes sociales, economía y empresa»

NUEVOS CANALES

\section{Fstrategia de marketing de medios sociales: conceptos y pasos para formar conexiones}

\section{Inma Rodríguez-Ardura}

Profesora de los Estudios de Economía y Empresa (UOC) y directora del Observatorio de la Nueva Economía (Internet Interdisciplinary Institute, UOC)

RESUMEN La emergencia de los medios sociales ha llevado a muchas empresas a adoptarlos como canales de marketing. Pero estos medios son lo suficientemente novedosos como para que muchos especialistas de marketing todavía se pregunten cómo planificar una estrategia de marketing de medios sociales efectiva, realmente orientada a implicar a los clientes potenciales. En este artículo se explica cómo dar forma a esta clase de estrategias de marketing digital, para lo que se muestran los conceptos y pasos clave a considerar en el proceso de planificación de estas estrategias, y cómo medir inmediatamente su impacto.

PALABRAS CLAVE medios sociales; marketing digital; estrategia de implicación

\section{Social Media Marketing Strategy: Concepts and Steps to Form Connections}

\begin{abstract}
The emergence of social media has led many companies to adopt them as marketing channels. Yet these media are novel enough to still leave many marketers unsure as to how to plan an effective social media marketing strategy, actually oriented towards engaging prospects. In this article, we discuss how to shape a social media strategy. To do so, we show the key concepts and steps involved in the planning process of this type of digital marketing strategy, and how to measure their impact immediately.
\end{abstract}

KEYWORDS social media; digital marketing; engagement strategy

\section{Introducción}

Los medios sociales son utilizados de manera cotidiana por muchas personas, que se sirven de ellos para colaborar, informarse, compartir opiniones y experiencias, o ampliar y consolidar sus redes personales de relaciones. 
Diariamente, miles de personas en todo el mundo contribuyen a proyectos colaborativos como Wikipedia, que en septiembre del 2014, solamente en sus ediciones en castellano e inglés, sumaba casi 6 millones de artículos (Wikipedia, 2014). Y se cuentan por millones los usuarios que consultan los contenidos resultantes de este inmenso trabajo colaborativo, lo que por esas mismas fechas hacía de Wikipedia el sexto sitio más visitado en Internet (Alexa, 2014). De modo análogo, miles de usuarios juegan en redes y mundos virtuales con otras personas; publican, comparten o consultan comentarios en Twitter y la blogosfera; y se sirven de comunidades de contenidos, como YouTube, Yelp, TripAdvisor, Instagram, Flickr, Pinterest, y otras muchas más para publicar, compartir o consultar vídeos, opiniones, fotografías, proyectos personales...Y cómo pasar por alto los millones de personas que se han hecho usuarios asiduos de sitios de redes sociales (Facebook, Tuenti, Linkedln, etc.). En ellos encuentran las plataformas adecuadas para compartir perfiles personales, encontrarse con otras personas y conversar.

Afirmar que las iniciativas de marketing en los medios sociales son imprescindibles es ya una obviedad. Pero ¿cómo aprovechar las enormes oportunidades que presentan estos medios para estrechar vínculos con los consumidores y contribuir a los objetivos de marketing? Dar respuesta a esa pregunta es, en cambio, harina de otro costal. Muchos esfuerzos de marketing en medios sociales son inconsistentes (Wilson et al., 2011) de modo que, en vez de responder a una estrategia de marketing digital bien diseñada y adecuadamente integrada, están disgregados o se guían por la única premisa de que cuanta más visibilidad y más conexiones mejor (Piskorski, 2011); es por ello por lo que no acaban siendo eficaces.

Como veremos a continuación, la estrategia de marketing de medios sociales no consiste únicamente en diseñar un perfil corporativo en medios sociales, y lanzar tuits, posts, fotos, vídeos o pins. Si bien estos son aspectos importantes, hay también otros elementos a tener en cuenta, como por ejemplo, el conocimiento de las características, hábitos y preferencias de los usuarios y potenciales clientes que utilizan los medios sociales, o el despliegue de programas que articulen adecuadamente contenidos generados por los usuarios con los de la propia marca (Rodríguez-Ardura, 2014). Se impone, en definitiva, la necesidad de diseñar una estrategia de marketing de medios sociales que esté bien integrada en los planes de marketing, como también de evaluar sus resultados de manera rápida y continuada.

\section{Medios sociales e implicación del consumidor}

¿A qué nos referimos al hablar de estrategia de marketing de medios sociales? El marketing de medios sociales comprende aquellas actividades de marketing digital con las que la marca se sirve de los medios sociales para ganar la colaboración de los consumidores e implicarlos en comunicaciones u otras iniciativas promovidas por la marca (Rodríguez-Ardura, 2014). Para ello recurre a métodos y tecnologías de observación, que permiten rastrear y analizar las conversaciones públicas de usuarios y consumidores en los medios sociales, y aprender de ellas. También promueve y desarrolla comunidades virtuales que giran alrededor de la marca; involucra a los consumidores y otros públicos de interés en iniciativas de innovación abierta; les ayuda, y les proporciona soporte a través de los medios sociales; y, en definitiva, se asegura de promover y facilitar un diálogo estrecho con los consumidores y usuarios de medios sociales, que contribuya a los objetivos de marketing.

La estrategia de marketing de medios sociales se concreta en una idea: ganar la implicación del consumidor (Rodríguez-Ardura et al., 2010). Y para ello, la marca se preocupa por conocer a fondo las características y comportamientos de los destinatarios de los esfuerzos de marketing, y por desarrollar contenidos y propuestas de valor que les faciliten experiencias entretenidas, útiles y participativas en los medios sociales.

Como, además, cada medio social tiene sus particularidades, conviene conocer bien las características de cada soporte o plataforma concreta de comunicación y los usos específicos que le dan sus usuarios. Esto puede llevar, por ejemplo, a utilizar blogs para proporcionar información en profundidad sobre los productos de la marca, o para acercar y hacer más humana la organización. Los blogs, además, pueden servir tanto para conectar con consumidores finales como con públicos profesionales. Twitter también puede ser muy efectivo para establecer vínculos con consumidores finales y con públicos profesionales, a la vez que permite enlazar con contenidos informativos y atractivos disponibles en otras plataformas en línea. Por su parte, Facebook puede ser muy adecuado para alcanzar a los consumidores finales, y es especialmente apropiado para difundir vídeos y contenidos 
entretenidos. Linkedln, en cambio, podrá utilizarse para conectar con públicos eminentemente profesionales, diff́ciles de contactar por otros canales, a los que se puede dar a conocerlos resultados de procesos de innovación para la marca, eventos corporativos, etc.

\section{Fases clave}

Si las plataformas sociales se utilizan como meros altavoces donde difundir mensajes comerciales, por muchos "amigos" y "seguidores" que se generen, difícilmente se podrá conseguir el deseado retorno de la inversión en estos medios. Porque, ¿a quién le apetece atender una propuesta de ventas cuando está entretenido, hablando con amigos y conocidos de su red social virtual? (Piskorski, 2011).

Para que la estrategia de marketing de medios sociales sea realmente efectiva, en primer lugar habrá que decidir sus objetivos de marketing, que además deberán estar en consonancia con los objetivos generales de negocio. También se tendrá que decidir cuál es el público o públicos objetivo al que se dirigirá el programa de marketing de medios sociales, lo que requerirá de un conocimiento previo de sus características y comportamientos (GIAMANCO y Gregoire, 2012).

Los objetivos que se proponga la estrategia de marketing de medios sociales pueden ser muy variados; desde establecer una verdadera conversación sobre nuevas variedades de productos para la marca, a proporcionar soporte al cliente que ya ha adquirido los productos. Por su parte, la definición del público objetivo lleva pareja el estudio en profundidad de sus características, sus preferencias, y sus hábitos en el uso de medios sociales.

En segundo lugar, y una vez determinados los objetivos de marketing y definido el público objetivo, habrá que identificar aquellos medios o plataformas sociales concretas que se utilizarán. Es poco probable que se opte por una única plataforma, ya que los consumidores y públicos de interés suelen utilizar más de una. Lo que habitualmente suele ser más conveniente, por lo tanto, es definir un mix de medios sociales, que a su vez se integre adecuadamente en el programa de comunicación de marketing de la organización.

Es deseable que los contenidos que luego se desarrollen para cada medio social sean "líquidos", es decir, fácilmente trasladables de una a otra plataforma (Draftfcb, 2013). Esto, sin embargo, no quiere decir que los contenidos deban replicarse de un medio a otro, pues en realidad se deberán ajustar a las características comunicativas de cada plataforma y a su audiencia particular. Pero sí que, en cambio, se podrán compartir algunas piezas comunicativas; y habrá que trabajar para asegurar que cada acción en medios sociales, a la vez que implique y fomente la conversación con los consumidores, haga crecer la historia y reputación de la marca, y contribuya a que los usuarios se formen una imagen sólida y coherente sobre ella.

En tercer lugar, convendrá definir el tipo de contenidos, y el esquema comunicativo que se utilizará para distribuir dichos contenidos. Conviene que en este tipo de decisiones se tengan en cuenta los objetivos de marketing que se han propuesto conseguir, los gustos y hábitos del público objetivo, el presupuesto de que se dispone y, cómo no, las características comunicativas de cada medio social.

Las decisiones sobre el esquema comunicativo son especialmente importantes. Aquí, más que generar propuestas comerciales y de ventas alrededor de la marca, conviene promover, estimular y facilitar información y recursos con valor para el consumidor. Y, en lugar de difundir contenidos de un modo prácticamente unidireccional, es mejor escuchar a los consumidores, "conectar" con ellos, y combinar los comentarios, recursos y contenidos que aportan con los recursos e informaciones propios, elaborados para la marca. Con todo ello se buscará dotar a la plataforma social de contenidos y recursos que, en su conjunto, aporten algo, sean útiles, entretengan, o proporcionen experiencias interesantes alrededor de la marca.

En cuarto lugar, habrá que desarrollar y gestionar eficazmente la distribución de contenidos. La presencia de la marca en medios sociales requiere de la preparación continuada de recursos en línea, así como del seguimiento, estímulo y promoción de las conversaciones o conexiones con los usuarios. Estas actividades pueden ser realizadas por el propio equipo de profesionales de la organización, si bien también cabe contar con la colaboración de agencias y firmas especializadas (Rodríguez-Ardura, 2014).

Cada nuevo post, tuit, vídeo o pin de la marca ofrece una oportunidad para generar tráfico de usuarios hacia el sitio web, las apps y los contenidos en medios sociales. De modo parecido, si los consumidores pueden 
transferir, citar y comentar fácilmente los contenidos de la marca a sus redes sociales virtuales, se conseguirá aumentar su notoriedad y visibilidad más fácilmente, lo que además mejorará el posicionamiento de los contenidos entre los resultados de los buscadores y, consecuentemente, generará más tráfico hacia ellos (Rodríguez-Ardura, 2014). Todo ello justifica un paso clave: la optimización de medios sociales o, lo que es lo mismo, el despliegue de iniciativas que faciliten la transferencia de contenidos entre medios sociales; y entre los medios sociales y el sitio y las apps de la marca.

\section{Evaluación inmediata de resultados}

La evaluación inmediata de los resultados de las iniciativas de marketing en medios sociales es esencial, porque proporciona información muy útil para avanzar y mejorar rápidamente en el desarrollo de una estrategia de medios sociales que realmente se gane la implicación del consumidor.

Los valores de los principales indicadores que cabe considerar muchas veces pueden ser medidos a partir de la información que proporcionan los propios medios sociales. En otros casos -como por ejemplo, en la medición de los ratios de conversión en usuarios y en clientes- cabe recurrir a técnicas de analítica web, que aportan información sobre la audiencia del sitio de la marca. En cualquier caso, cabe destacar los siguientes indicadores (Rodríguez-Ardura, 2014):

- Implicación con la marca (Hoffman y Fodor, 2010): número de seguidores de la marca en Twitter; número de "me gusta" en Facebook, Instagram o Pinterest; número de visualizaciones de fotos o vídeos en Instagram, Flickr, YouTube, etc.

- Conversación con la marca: número de comentarios generados en promedio por los posts, tuits, imágenes, vídeos o pins sobre la marca en cada medio social.

- Amplificación de la marca: número de ocasiones que, en promedio, los posts de la marca son reblogeados, retuiteados, compartidos o repineados en cada medio social (Sable Lopez, 2013).

- Conversión en usuarios: proporción de nuevos usuarios del sitio procedentes de cada medio social (SimplyMeasured, 2013), frecuencia media de las visitas al sitio por seguidores de cada medio social, etc.

- Conversión en clientes: proporción de seguidores de cada medio social que adquieren productos en el sitio (SimplyMeasured, 2013).

\section{Conclusiones}

En la medida en que los medios sociales se han hecho con un hueco importante en la vida de los consumidores, las marcas no tienen más opción que desarrollar estrategias apropiadas de marketing de medios sociales para mantener conexiones estrechas con sus clientes, actuales o potenciales. Es bueno empezar cuando antes, aunque para ello haya que recurrir a iniciativas de pequeño alcance. Pero también lo es que estas iniciativas partan de un conocimiento certero sobre el comportamiento de los consumidores, estén sólidamente integradas en la estrategia de marketing, y sean el resultado de un cuidadoso proceso de planificación.

Asimismo, conviene no perder de vista que las estrategias de marketing de medios sociales se han de ir mejorando a partir del conocimiento acumulado sobre sus resultados, y los cambios que se detecten en el entorno. Wikipedia, Facebook y Twitter tienen, respectivamente, tan solo 13, 10 y 8 años de vida, así que conviene estar preparado para todo lo que pueda venir a continuación.

\section{Bibliografía}

(2014). «Wikipedia statistics: article count (official)» [documento en línea], Wikipedia. <http://stats. wikimedia.org/ EN/TablesArticlesTotal.htm> 
ALEXA (2014). Alexa traffic Rank [documento en línea]. <http://www.alexa.com/>

DRAFTFCB (2013). «Transmedia storytelling» [documento en línea]. <https://www.youtube.com/ watch?v=TloOYKzIFEg>

GIAMANCO, B.; GREGOIRE, K. (2012). «Tweet me, friend me, make me buy». Harvard Business Review. Vol. 90, núm. 7/8, págs. 88-93.

Hoffman, D.L.; Fodor, M. (2010). «Can you measure the ROI of your social media marketing?». Sloan Management Review. Vol. 52, núm. 1, págs. 41-49.

Piskorski, M. (2011). «Social strategies that work». Harvard Business Review. Vol. 89, núm.11, págs. 116-122.

Rodríguez-Ardura, i. (2014). Marketing digital y comercio electrónico. Madrid: Pirámide.

RODRÍGUEZ-ARDURA, I.; MARTíNEZ-LÓPEZ, F.J.; LUNA, P. (2010). "Going with the consumer towards the social web environment: a review of extant knowledge». International Journal of Electronic Marketing and Retailing. Vol. 3, núm. 4, págs. 415-440.

SABLE LOPEZ, J. (2013). «Social engagement metrics that matter - Measuring, tracking, and reporting FTW» [documento en línea], The Moz Blog, 13 de febrero. <http://moz.com/blog>

SIMPLY MEASURED (2013). «5 social media metrics that your business should be tracking» [documento en línea], 29 de octubre. <http://thenextweb.com>

WILSON, H.J.; GUINAN, P.J.; SALVATORE, P.; WEINBERG, B.D. (2011). «What's your social media strategy?». Harvard Business Review. Vol. 89, núm. 7/8, págs. 23-25.

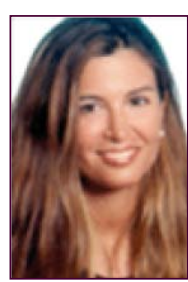

\section{Inma Rodríguez-Ardura \\ irodriguez@uoc.edu \\ Profesora de los Estudios de Economía y Empresa (UOC) y directora del Observatorio de la Nueva Economía (Internet Interdisciplinary Institute, UOC)}

Profesora agregada en Comercialización e Investigación de Mercados de los Estudios de Economía y Empresa de la UOC y directora del Observatorio de la Nueva Economía, grupo de investigación del Internet Interdisciplinary Institute de la UOC -reconocido como Grupo de Investigación Consolidado por la Generalitat de Cataluña. La Dra. Rodríguez-Ardura ejerce como profesora de las titulaciones de Economía y Empresa, y del máster universitario y el doctorado de Sociedad de la Información y el Conocimiento de la UOC. Ha sido profesora asociada de la Universidad de Barcelona, Visiting Fellow de la Universidad de Oxford, y Visiting Professor de Babson College, Boston. En la UOC creó y ha sido directora de las titulaciones de grado y posgrado de Marketing e Investigación de Mercados; y directora del máster universitario, y el Programa de Doctorado en Sociedad de la Información y el Conocimiento. Asimismo, desarrolla proyectos de investigación competitiva financiados por instituciones europeas e internacionales. Los resultados de sus investigaciones han sido publicados en múltiples revistas científicas e internacionales de impacto. También es autora de diversos libros en los campos del marketing, la comunicación y el comercio electrónico. Su obra más reciente es Marketing digital y comercio electrónico (Pirámide, 2014).

Los textos publicados en esta revista están -si no se indica lo contrario- bajo una licencia Reconocimiento-Sin obras derivadas 3.0 España de Creative Commons. Puede copiarlos, distribuirlos y comunicarlos públicamente siempre que cite su autor y la revista y la institución que los publica (autoría, nombre de la revista, institución editora); no haga con ellos obras derivadas. La licencia completa se puede consultar en http://creativecommons.org/licenses/by-nd/3.0/es/deed.es.

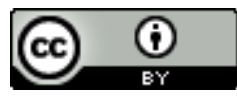

